Case Reports
in Dermatology
Case Rep Dermatol 2021;13:62-68

DOI: 10.1159/000511495

Published online: February 1, 2021 (c) 2021 The Author(s)

Published by S. Karger AG, Basel www.karger.com/cde

\title{
Papulopustular and Ocular Rosacea with an Alleged Coincidence of Cutaneous Lupus Erythematosus: A Case Report
}

\author{
Irma Bernadette S. Sitohang ${ }^{\mathrm{a}}$ Firman Parrol $^{\mathrm{a}} \quad$ Eyleny Meisyah Fitri ${ }^{\mathrm{a}}$ \\ Rina La Distia Norab \\ aDepartment of Dermatology and Venereology, Faculty of Medicine, Universitas \\ Indonesia, dr. Cipto Mangunkusumo Hospital, Jakarta, Indonesia; ${ }^{b}$ Department of \\ Ophthalmology, Faculty of Medicine, Universitas Indonesia, dr. Cipto Mangunkusumo \\ Hospital, Jakarta, Indonesia
}

\section{Keywords}

Case report $\cdot$ Papulopustular rosacea $\cdot$ Ocular rosacea $\cdot$ Cutaneous lupus erythematosus

\begin{abstract}
Rosacea is a chronic inflammatory skin disease characterized by central facial erythema with or without ocular involvement. It is often difficult to distinguish rosacea from other malar rashes, one of which is acute cutaneous lupus erythematosus (CLE), particularly when there is an increase in antinuclear antibody (ANA) level. We report the case of a 16-year old woman with facial erythematous plaque accompanied by papules and pustules, reddened eyes, and swollen eyelids since the last one year. Dermoscopic examination revealed telangiectasia, and skin scraping examination with $20 \%$ potassium hydroxide identified the presence of Demodex folliculorum. Further ocular examination also revealed blepharitis, dysfunction of Meibomian gland, cicatrix, and corneal neovascularization. The ANA titer was positive (1:320), while the anti-dsDNA was negative. The patient was treated according to standard treatment for rosacea. The patient showed a satisfactory response following 2 weeks of therapy. Signs of recurring red patches with papules, pustules, telangiectasia, and identification of $D$. folliculorum on skin scraping examination led to the diagnosis of papulopustular rosacea. A positive
\end{abstract}

Irma Bernadette S. Sitohang
Department of Dermatology and Venereology
Faculty of Medicine, Universitas Indonesia, dr. Cipto Mangunkusumo Hospital
Jalan Salemba No. 6, Jakarta 10430 (Indonesia)
irma_bernadette@yahoo.com




\section{Case Reports in Dermatology}

Sitohang et al.: Papulopustular and Ocular Rosacea with Coincidence of CLE

ANA test may also be present in other diseases, e.g. acute CLE. Therefore, the diagnosis of rosacea remains a challenge. Thorough observation and examination must be done in order to yield an accurate diagnosis of rosacea.

\section{Introduction}

Rosacea is a chronic inflammatory disease of the skin, characterized by central facial erythema. Rosacea might be accompanied by ocular involvement, and its symptoms often get worsened by sun exposure and various external factors. The pathogenesis of rosacea has not yet been fully elucidated $[1,2]$. It generally affects individuals in their third or fourth decade of life, with a tendency to affect a greater proportion of women than men (3:1) [3]. Establishing the diagnosis of rosacea greatly depends on clinical manifestations since it has neither specific histopathological findings nor diagnostic serological tests [4]. Clinical manifestations of rosacea may mimic other facial skin disorders, one of which is acute cutaneous lupus erythematosus (CLE). Therefore, the diagnosis of rosacea often becomes a challenge for physicians. Rosacea and acute CLE show many similarities in terms of clinical manifestation, including the tendency to affect greater proportion of women, central facial erythema, and aggravated symptoms following sun exposure [2,3]. In this case report, we present a case of papulopustular and ocular rosacea with an alleged coincidence of CLE.

\section{Case Report/Case Presentation}

A 16-year-old woman was referred to the dermatology clinic with pustules and erythema on her face since one year. The pustule initially appeared on both cheeks and later extended to the entire face accompanied by pain and itch (Fig. 1a-c). The lesion became more noticeable following sun exposure and spicy food consumption. The patient had previously been treated, and the treatment response was initially good, but the symptoms recurred. The patient's right eye became red and sore. She also became more sensitive to light since the last few months. There was no history of fever, hair loss, dandruff, mouth ulcers, headaches, coughing/shortness of breath, abdominal pain, and genitourinary disorders. Dermatological examination on both cheeks and nasal revealed multiple scattered-to-concentrated erythematous lesions with lenticular-to-plaque size and well-defined border, also erythematous papules and pustules in several places (Fig. 2a-c). Ocular examination showed conjunctivitis with periorbital edema (shown in Fig. 2d). Dermoscopic examination of the cheek showed telangiectasia (Fig. 3a). Demodex folliculorum was identified on skin scraping examination with $20 \%$ potassium hydroxide (Fig. 3b). Complete blood count, white blood cell differential count, and kidney function tests were within normal limits. Antinuclear antibody (ANA) test yield positive results with a titer of 1:320 showing a centriole and rough speckled pattern, while anti-dsDNA level was within normal limits $(<10 \mathrm{IU} / \mathrm{mL}$ ). C3 and C4 levels were also within normal limits (89.8 and $20.2 \mathrm{mg} / \mathrm{dL}$, respectively; normal range: $85-160$ and $10-40 \mathrm{mg} / \mathrm{dL}$, respectively). The patient was diagnosed with papulopustular and ocular rosacea with suspected coincidence of acute CLE.

The patient was prescribed sunscreen with sun protection factor (SPF) 45 to be applied every $3 \mathrm{~h}, 1 \%$ metronidazole in $20 \mathrm{~g}$ of hypoallergenic ambiphilic cream to be applied every

\section{Karger'=}




\section{Case Reports in Dermatology}

Case Rep Dermatol 2021;13:62-68

DOI: 10.1159/000511495

morning and evening, and adapalene night cream. In Indonesia, metronidazole is not available in topical cream preparations. Consequently, it is produced independently by the hospital pharmacy with an individual dosage. The patient was also treated with $100 \mathrm{mg}$ of doxycycline bid, fluorometholone eye drops, terramycin ointment, and artificial tears for both eyes.

Following 2 weeks of therapy, the patient showed an improvement of the lesions on both cheeks and nasal. The number of papules and pustules and the degree of erythema were also significantly reduced (Fig. 4a-c), and we also identified a remarkable improvement of the ocular abnormalities (Fig. 4d).

\section{Discussion}

Facial erythema along with ocular abnormalities led to the diagnosis of papulopustular and ocular rosacea. There are four main subtypes of rosacea, which are erythematotelangiectatic rosacea, papulopustular rosacea, phymatous rosacea, and ocular rosacea. Nevertheless, any combination of rosacea subtypes may also occur. In 2002, National Rosacea Society established clinical criteria for the diagnosis of rosacea. The primary criteria include the presence of transient erythema (flushing), persistent erythema, papules, pustules, and/or telangiectasia $[5,6]$. The presence of at least one of these signs along with a centrally distributed facial lesions lead to the diagnosis of rosacea. The secondary criteria include burning or stinging sensation, elevated reddish plaque, dry skin, edema, ocular manifestations, peripheral lesions, and phymatous changes [5]. These secondary signs often accompany the primary signs [2,4].

The primary criteria found in our patient were persistent erythema, papules, pustules, and telangiectasia, while the only secondary criterion found was ocular manifestations. Pustular lesions and telangiectasia tend to lead to the diagnosis of papulopustular rosacea. Also, the telangiectasia of rosacea can be differentiated from that of CLE since the telangiectasia of CLE is commonly found on lesions which have already been treated with topical steroids. In conclusion, the characteristics of telangiectasia in this patient are more representative of rosacea [7].

The diagnosis of ocular rosacea is established by the presence of at least two of these clinical signs, including facial symptoms of rosacea, conjunctival or eyelid disorders, posterior blepharitis with conjunctival hyperemia, follicular and papillary conjunctivitis with or without scars, corneal disease, marginal ulcers with perforation, pseudo-pterygium or corneal neovascularization, and the presence of infiltrates or scars $[8,9]$. In this patient, the diagnosis of ocular rosacea is established based on the findings of physical examination conducted by the ophthalmologist; they included meibomian gland disorders, conjunctivitis, corneal scars, and corneal neovascularization.

A positive ANA test led to the diagnosis of acute CLE. CLE is reported in $20-60 \%$ of patients with systemic lupus erythematosus (SLE) which most commonly presents with systemic abnormalities [3]. Nevertheless, the clinical manifestations in this patient did not meet the new classification criteria for SLE jointly supported by the European League Against Rheumatism (EULAR) and the American College of Rheumatology (ACR). The 2019 EULAR/ACR classification criteria for SLE include positive ANA (titer of $\geq 1: 80$ on HEp- 2 cells or an equivalent positive test) at least once as obligatory entry criterion; followed by additive weighted criteria grouped in 7 clinical (constitutional, hematological, neuropsychiatric, mucocutaneous, serosal, musculoskeletal, renal) and 3 immunological (antiphospholipid antibodies, complement proteins, SLE-specific antibodies) domains, and weighted from 2 to 10. Patients

\section{Karger'=}




\section{Case Reports in Dermatology}

Case Rep Dermatol 2021;13:62-68

DOI: 10.1159/000511495 (c) 2021 The Author(s). Published by S. Karger AG, Basel www.karger.com/cde

Sitohang et al.: Papulopustular and Ocular Rosacea with Coincidence of CLE

accumulating $\geq 10$ points are classified. This patient only met entry criterion and did not fulfill the clinical and immunological domains and criteria (score 0) [10]. The positive ANA test (1:320) caused a diagnostic dilemma in the diagnosis of rosacea since both rosacea and CLE might have similar clinical manifestations. However, there are several studies reporting significantly greater proportion of rosacea cases with positive ANA test compared to those with negative ANA test.

In 2013, Woźniacka et al. [4] reported that $53.5 \%$ of rosacea patients had an ANA titer of $\geq 1: 160$, comprising $13.86 \%$ of patients with a titer of $1: 320,8.91 \%$ of patients with a titer of $1: 640$, and $6.93 \%$ of patients with a titer of $\geq 1: 1,280$. In the aforementioned study, only 2 out of $26(7.7 \%)$ healthy individuals had increased ANA titers (1:160 and 1:320). Two years following the initial investigation, the study found that there was no patient with an ANA titer of $>1: 640$ developing autoimmune disease. These findings indicated that the ANA titer could not be used as a reference in differentiating rosacea and acute CLE although an ANA titer of $\geq 1: 80$ is required for the diagnosis of SLE $[10,11]$. Despite being diagnostic criteria for SLE, the specificity of high ANA titers is unknown [4]. The ANA test provides high diagnostic sensitivity in the cases of acute CLE with SLE, while the anti-dsDNA and Sm test provides high diagnostic specificity [4]. Furthermore, ANA titers are found to increase in several conditions other than autoimmune diseases, e.g. drug-induced ANAs, ischemic stroke, coronary artery diseases, infectious diseases, and cancers, none of which was identified in our case [4]. Therefore, the increased ANA titers should al ways be considered for the diagnosis of an autoimmune disease.

The patient was given the treatment regimen for rosacea, which consisted of $1 \%$ metronidazole in 20 mg hypoallergenic ambiphilic cream, sunscreen with SPF 45, 0.1\% adapalene cream, and doxycycline. Following the therapy, the patient showed satisfactory improvement. Metronidazole and doxycycline are two recommended therapies by the Food and Drug Administration (FDA) for the treatment of rosacea. An in vitro study showed that metronidazole minimizes the degree of inflammation by inhibiting inflammatory mediators produced by neutrophils also significantly reduces the damage caused by oxidative stress. The palmitoleic acid in human skin is found to enhance this metronidazole's effects. Reported as ineffective against $D$. folliculorum, metronidazole is found efficacious in rosacea due to its potent antiinflammatory properties [12].

Adapalene is a naphthoic acid derivative, acting as a potent agonist of retinoic acid receptor, which possesses anti-inflammatory and anti-proliferative properties with remarkable follicular penetration. Ertl et al. [13] reported significant reduction of papules, pustules, and erythema following the use of adapalene cream for the treatment of rosacea. Adapalene is also shown to combat photo-aging, the manifestations of which, e.g. elastic degeneration, can also be identified in rosacea $[14,15]$. Doxycycline exhibits anti-inflammatory and immunomodulating properties, also helps reducing vasodilation in rosacea. Doxycycline inhibits phospholipase A2, nitric oxide synthetase expression, and mitogen-induced human lymphocytic proliferation, also accelerates the nitric oxide synthetase degradation, which explains the potent anti-inflammatory effects. As for the reduction of vasodilation, doxycycline inhibits gelatinase $\mathrm{A}$ and $\mathrm{B}$ which subsequently promotes the integrity of capillary wall and connective tissues, decreases sensitivity to vasodilatory stimuli, and downregulates pro-inflammatory cytokines [16]. A study showed improvement of skin and ocular lesions in patients with rosacea following the administration of $100 \mathrm{mg}$ doxycycline b.i.d. for 2 weeks along with avoidance of aggravating factors.

SLE is the prototypic multisystem autoimmune disorder with a broad spectrum of clinical presentations encompassing almost all organs and tissues. It is a chronic disease of variable

\section{Karger'=}




\section{Case Reports in Dermatology}

Sitohang et al.: Papulopustular and Ocular Rosacea with Coincidence of CLE

severity with a waxing and waning course, with significant morbidity that can be fatal - if not treated early. The disease starts with a preclinical phase characterized by autoantibodies common to other systemic autoimmune diseases and proceeds with a more disease-specific clinically overt autoimmune phase [11]. Hence, further observation and collaboration with a rheumatologist will be sought when the rosacea has been controlled in order to further assess the possibility of autoimmune diseases in this patient.

This case report presents the evaluation of the essential findings on papulopustular rosacea. As there are a lot of differential diagnoses for facial lesions such as in this case, astute clinical judgment is necessary in establishing the diagnosis of rosacea. All similarities and differences with the alleged coincidence of autoimmune disease were reviewed and discussed by means of current references and consensus. It is hoped that this case report can serve as a basis for a better diagnostic approach of rosacea.

\section{Conclusion}

Establishing the diagnosis of a malar rash is challenging because of its numerous possible causes. Furthermore, the finding of an ANA titer of $>1: 80$ raised the suspicion of autoimmune diseases. Long-term observation is needed to detect any autoimmune diseases that can develop. A comprehensive diagnostic approach and careful exclusion of any differential diagnoses are crucial for better outcome.

\section{Statement of Ethics}

A written photograph publication consent form was obtained. The study was conducted ethically in accordance with the World Medical Association Declaration of Helsinki.

\section{Conflict of Interest Statement}

The authors declare no conflicts of interest exist related to this work.

\section{Funding Sources}

There is no funding source that supported the work.

\section{Author Contributions}

Each person named as an author has made substantial contribution to the paper. I.B.S.S. conceived and designed the work, wrote the initial draft of the manuscript and interpreted the data, revised it critically for important intellectual content, was responsible for communication with the journal during the manuscript submission, peer review, and publication. F.P. assisted in the preparation of the manuscript. All authors approved the final version of the

\section{Karger'=}




\section{Case Reports in Dermatology}

\begin{tabular}{l|l}
\hline Case Rep Dermatol 2021;13:62-68 \\
\hline DOI: 10.1159/000511495 & $\begin{array}{l}\text { @ 2021 The Author(s). Published by S. Karger AG, Basel } \\
\text { www.karger.com/cde }\end{array}$ \\
\hline
\end{tabular}

Sitohang et al.: Papulopustular and Ocular Rosacea with Coincidence of CLE

manuscript and take responsibility for all aspects of the work in ensuring that questions related to the accuracy or integrity of any part of the work are appropriately answered and resolved.

\section{References}

1 Tan J, Almeida LM, Bewley A, Cribier B, Dlova NC, Gallo R, et al. Updating the diagnosis, classification and assessment of rosacea: recommendations from the global ROSacea COnsensus (ROSCO) panel. Br J Dermatol. 2017 Feb;176(2):431-8.

2 Marai MH. Clinico-pathological investigation of rosacea with particular regard to systemic diseases. Leeds: The University of Leeds; 2015.

3 Steinhoff M, Buddenkotte J. Rosacea. In: Goldsmith LA, Katz SI, Gilchrest BA, Paller AS, Leffell DJ, Wolff K, editors. Fitzpatrick's dermatology in general medicine. 9th ed. USA: McGraw-Hill; 2019. pp. 1419-47.

4 Woźniacka A, Salamon M, McCauliffe D, Sysa-Jędrzejowska A. Antinuclear antibodies in rosacea patients. Postepy Dermatol Alergol. 2013 Feb;30(1):1-5.

5 Wilkin J, Dahl M, Detmar M, Drake L, Feinstein A, Odom R, et al. Standard classification of rosacea: Report of the National Rosacea Society Expert Committee on the Classification and Staging of Rosacea. J Am Acad Dermatol. 2002 Apr;46(4):584-7.

6 Powell FC. Clinical practice. Rosacea. N Engl J Med. 2005 Feb;352(8):793-803.

7 Lallas A, Giacomel J, Argenziano G, García-García B, González-Fernández D, Zalaudek I, et al. Dermoscopy in general dermatology: practical tips for the clinician. Br J Dermatol. 2014 Mar;170(3):514-26.

8 Deepika D, Chintan M, Arun KJ. Ocular rosacea - A review. US Ophthalmic Rev. 2017;10(02):113-8.

9 Vieira AC, Höfling-Lima AL, Mannis MJ. Ocular rosacea-a review. Arq Bras Oftalmol. 2012 Oct;75(5):363-9.

10 Fanouriakis A, Kostopoulou M, Alunno A, Aringer M, Bajema I, Boletis JN, et al. 2019 update of the EULAR recommendations for the management of systemic lupus erythematosus. Ann Rheum Dis. 2019 Jun;78(6):736-45.

11 Bertsias G, Cervera R, Boumpas DT. Systemic lupus erythematosus: pathogenesis and clinical features. In: EULAR Textbook on Rheumatic Diseases. Kilchberg: EULAR; 2012. p. 476-505.

12 Hansen CB, Sombetrie RD. Diagnostic criteria for cutaneous lupus erythematosus. In: Maibach HI, Gorouhi F, editors. Evidence Based Dermatology. Shelton: People Medical Publishing House-USA; 2011. pp. 65-71.

13 Ertl GA, Levine NL, Kligman AM. A comparison of the efficacy of topical tretinoin and low-dose oral isotretinoin in rosacea. Arch Dermatol. 1994 Mar;130(3):319-24.

14 McClellan KJ, Noble S. Topical metronidazole. A review of its use in rosacea. Am J Clin Dermatol. 2000 MayJun;1(3):191-9.

15 Altinyazar HC, Koca R, Tekin NS, Eştürk E. Adapalene vs. metronidazole gel for the treatment of rosacea. Int J Dermatol. 2005 Mar;44(3):252-5.

16 Valentín S, Morales A, Sánchez JL, Rivera A. Safety and efficacy of doxycycline in the treatment of rosacea. Clin Cosmet Investig Dermatol. 2009 Aug;2:129-40.
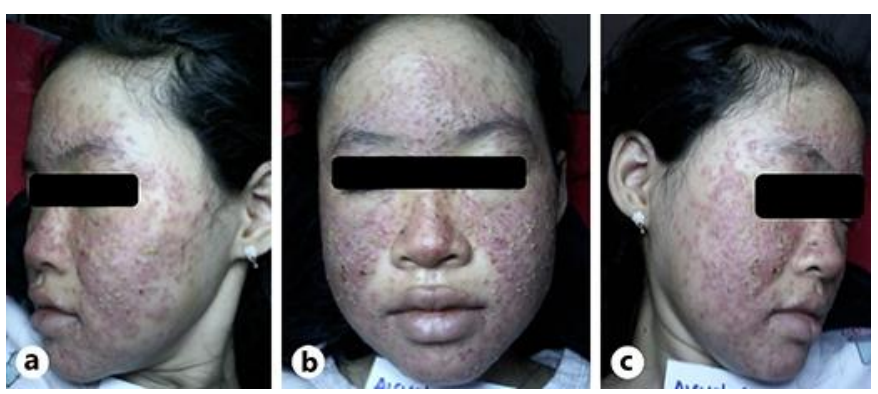

Fig. 1. a-c Early lesion: erythematous papules and multiple pustules on the face 1 year before.

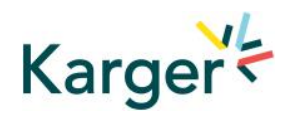




\section{Case Reports in Dermatology}
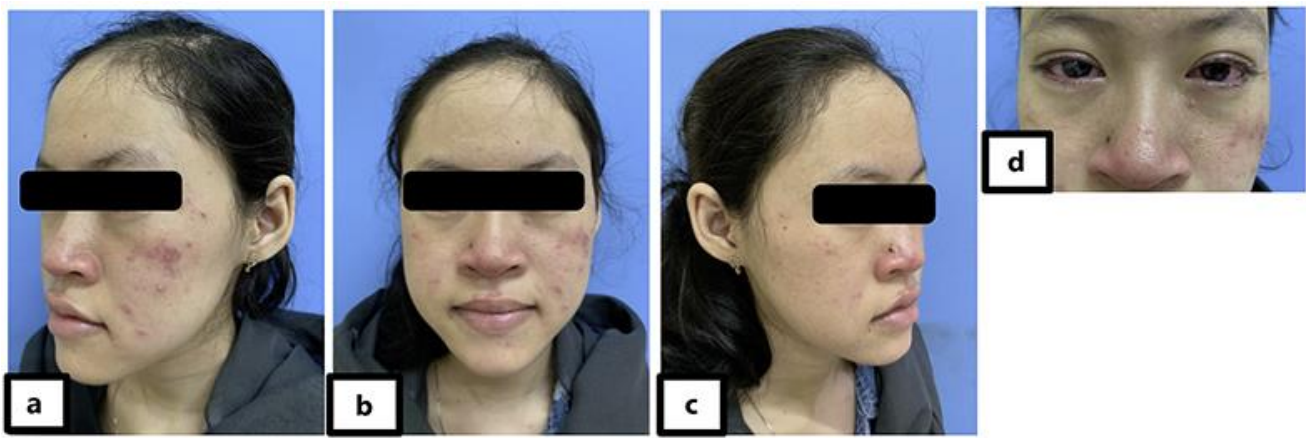

Fig. 2. a-c Erythematous papules and multiple pustules at the first visit. $\mathbf{d}$ Conjunctivitis and periorbital edema.

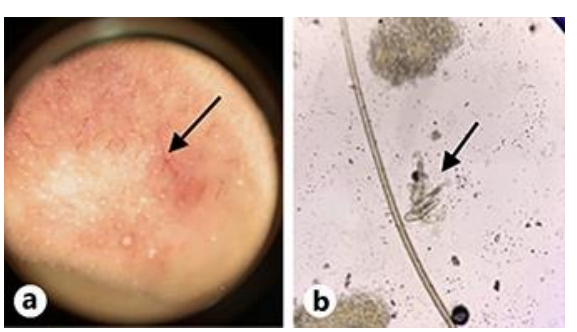

Fig. 3. a Dermoscopic examination of the cheek showing telangiectasia. $\mathbf{b}$ Demodex folliculorum on the skin scrapping test with $20 \%$ potassium hydroxide.
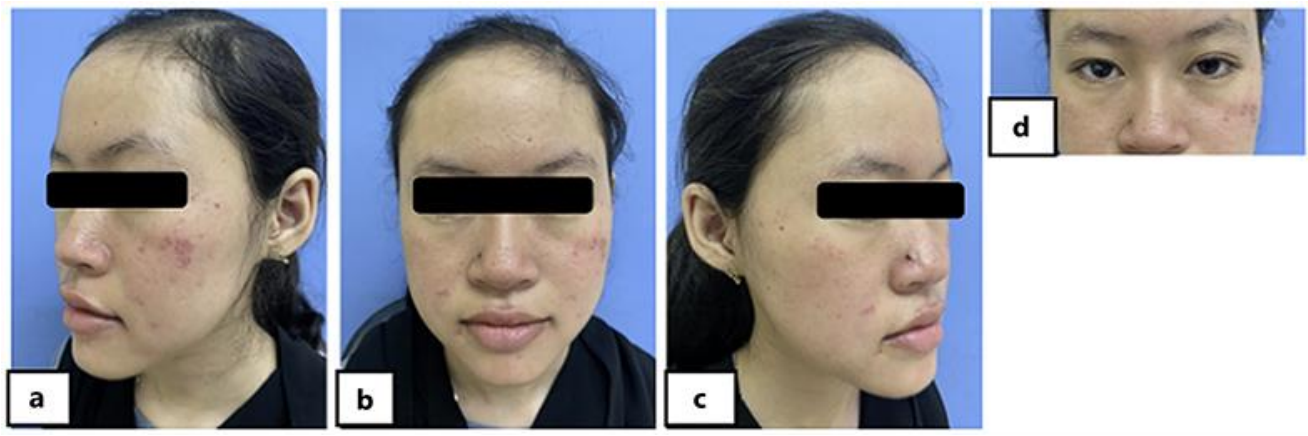

Fig. 4. a-c Improvement of facial lesions following 2 weeks of therapy. $\mathbf{d}$ Improvement of conjunctivitis and periorbital edema following 2 weeks of therapy. 